\title{
Spiral-like star-forming patterns in CALIFA early-type galaxies ${ }^{\star}$
}

\author{
J. M. Gomes ${ }^{1}$, P. Papaderos ${ }^{1}$, J. M. Vílchez ${ }^{2}$, C. Kehrig ${ }^{2}$, J. Iglesias-Páramo ${ }^{2,3}$, I. Breda ${ }^{1}$, M. D. Lehnert ${ }^{4}$,
} S. F. Sánchez ${ }^{5}$, B. Ziegler ${ }^{6}$, S. N. dos Reis ${ }^{1}$, J. Bland-Hawthorn ${ }^{7}$, L. Galbany ${ }^{8,9}$, D. J. Bomans ${ }^{10,11}$, F. F. Rosales-Ortega ${ }^{12}$, C. J. Walcher ${ }^{13}$, R. García-Benito ${ }^{2}$, I. Márquez ${ }^{2}$, A. del Olmo ${ }^{2}$, M. Mollá ${ }^{14}$, R. A. Marino ${ }^{15,16}$, C. Catalán-Torrecilla ${ }^{17}$, R. M. González Delgado ${ }^{2}$, Á. R. López-Sánchez ${ }^{18,19}$, and the CALIFA Collaboration

1 Instituto de Astrofîsica e Ciências do Espaço, Universidade do Porto, Centro de Astrofísica da Universidade do Porto, Rua das Estrelas, 4150-762 Porto, Portugal e-mail: jean@astro.up.pt

2 Instituto de Astrofísica de Andalucía (CSIC), Glorieta de la Astronomía s/n Aptdo. 3004, 18080 Granada, Spain

3 Estación Experimental de Zonas Aridas (CSIC), Ctra. de Sacramento s.n., La Cañada, 04120 Almerìa, Spain

4 Institut d'Astrophysique de Paris, UMR 7095, CNRS, Université Pierre et Marie Curie, 98 bis boulevard Arago, 75014 Paris, France

5 Instituto de Astronomía, Universidad Nacional Autonóma de Mexico, A.P. 70-264, 04510 México, D.F., Mexico

${ }^{6}$ University of Vienna, Türkenschanzstrasse 17, 1180 Vienna, Austria

7 Sydney Institute for Astronomy, University of Sydney, NSW 2006, Australia

8 Millennium Institute of Astrophysics, Chile

9 Departamento de Astronomía, Universidad de Chile, Casilla 36-D, 1058 Santiago, Chile

10 Astronomical Institute of the Ruhr-University Bochum, Universitätsstr. 150, 44580 Bochum, Germany

11 RUB Research Department "Plasmas with Complex Interactions”, Universitätsstr. 150, 44580 Bochum, Germany

12 Instituto Nacional de Astrofísica, Óptica y Electrónica, Luis E. Erro 1, 72840 Tonantzintla, Puebla, Mexico

13 Leibniz-Institut für Astrophysik Potsdam (AIP), An der Sternwarte 16, 14482 Potsdam, Germany

14 CIEMAT, Avda. Complutense 40, 28040 Madrid, Spain

15 CEI Campus Moncloa, UCM-UPM, Departamento de Astrofísica y CC. de la Atmósfera, Facultad de CC. Físicas, Universidad Complutense de Madrid, Avda. Complutense s/n, 28040 Madrid, Spain

16 Department of Physics, Institute for Astronomy, ETH Zürich, 8093 Zürich, Switzerland

17 Departamento de Astrofísica y CC. de la Atmósfera, Universidad Complutense de Madrid, 28040 Madrid, Spain

18 Australian Astronomical Observatory, PO Box 915, North Ryde, NSW 1670, Australia

19 Department of Physics and Astronomy, Macquarie University, NSW 2109, Australia

Received 26 February 2015 / Accepted 25 September 2015

\begin{abstract}
Based on a combined analysis of SDSS imaging and CALIFA integral field spectroscopy data, we report on the detection of faint $(24<$ $\left.\mu_{r} \mathrm{mag} / \square^{\prime \prime}<26\right)$ star-forming spiral-arm-like features in the periphery of three nearby early-type galaxies (ETGs). These features are of considerable interest because they document the still ongoing inside-out growth of some local ETGs and may add valuable observational insight into the origin and evolution of spiral structure in triaxial stellar systems. A characteristic property of the nebular component in the studied ETGs, classified $\mathrm{i}+$, is a two-radial-zone structure, with the inner zone that displays faint $(E W(\mathrm{H} \alpha) \simeq 1 \AA)$ low-ionization nuclear emission-line region (LINER) properties, and the outer one $(3 \AA<E W(\mathrm{H} \alpha) \leqslant 20 \AA)$ HII-region characteristics. This spatial segregation of nebular emission in two physically distinct concentric zones calls for an examination of aperture effects in studies of type i+ ETGs with single-fiber spectroscopic data.
\end{abstract}

Key words. galaxies: elliptical and lenticular, $\mathrm{cD}$ - galaxies: nuclei - galaxies: ISM - galaxies: kinematics and dynamics galaxies: star formation

\section{Introduction}

Studies of low-level star formation (SF) activity in early-type galaxies (ETGs) are fundamental for advancing our understanding of the build-up history and spectrophotometric evolution of these systems and elucidating the nature of the dominant excitation source of their warm interstellar medium (wim). Particularly valuable in this respect are observational insights into the spatial characteristics and temporal evolution of SF at

* Based on observations collected at the Centro Astronómico Hispano Alemán (CAHA) at Calar Alto, operated jointly by the MaxPlanck-Institut für Astronomie (MPIA) and the Instituto de Astrofísica de Andalucía (CSIC). the morphological interface between lenticulars (S0) and ellipticals (E), since they can offer key constraints on the origin of quenching (or, conversely, rejuvenation) in ETGs, and its implications on the spectrophotometric evolution of their structural components.

The origin of faint nebular emission in ETGs - long revealed through single-aperture and longslit spectroscopy (e.g., Phillips et al. 1986; Demoulin-Ulrich et al. 1984; Kim 1989; Trinchieri \& di Serego Alighieri 1991; Annibali et al. 2010; Yan \& Blanton 2012), narrow-band imaging (e.g., Finkelman et al. 2010; Kulkarni et al. 2014), and more comprehensively, with integral field spectroscopy (IFS, e.g., Sarzi et al. 2006, 2010; Kehrig et al. 2012; Papaderos et al. 2013; Singh et al. 2013) - 
continues to provide fodder for an intense ongoing debate. Low-level SF activity (see, e.g., Trager et al. 2000; Schawinski et al. 2007; Shapiro et al. 2010, and references therein) offers an appealing hypothesis for the excitation of the extended (extranuclear) wim in ETGs, as an alternative or supplementary mechanism to photoionization by an active galactic nucleus (AGN, e.g., Ho 2008) or the evolved ( $\geq 10^{8} \mathrm{yr}$ ) post-AGB (pAGB) population (e.g., Trinchieri \& di Serego Alighieri 1991; Binette et al. 1994; Stasińska et al. 2008), or excitation by fast shocks (e.g., Dopita \& Sutherland 1995). Observational evidence for SF in ETGs comes from combined near-UV, SDSS $r$ and mid-IR color studies (e.g., Kaviraj et al. 2007; Gil de Paz et al. 2007; Ko et al. $2014)$, with $10-15 \%$ of the stellar mass $\left(\mathcal{M}_{\star}\right)$ in these systems assembling in a declining SF process since $z \simeq 1$ (Kaviraj et al. 2008). The presence of a small fraction $(\sim 5.7 \%)$ of blue ETGs in the local Universe with estimated star-formation rates (SFRs) between 0.5 and $50 M_{\odot} / y r$ (Schawinski et al. 2009) lends additional support to this notion. That an appreciable fraction of nearby ETGs continues its build-up at a very slow pace, with specific SFRs of $\lesssim 10^{-11} \mathrm{yr}^{-1}$ (e.g., Salim et al. 2012), is further indicated by multiwavelength studies that combine singlefiber spectroscopy with GALEX/SDSS photometry (e.g., Huang \& Gu 2009; Petty et al. 2013; Pan et al. 2014).

However, our knowledge on the spatial distribution of SF in ETGs is still fragmentary, even though a few detailed studies of individual systems or small galaxy samples seem to unequivocally point to an increasing luminosity contribution from youngto-intermediate-age stellar populations towards their periphery. This is suggested by, e.g., UV-optical color profiles of bluish ETGs at the boundary of the green valley, which displays red centers and blue outskirts (Fang et al. 2012). UV-bright shells and in some cases striking spiral arms in the extranuclear component of some nearby ETGs (e.g., Rampazzo et al. 2007; Salim \& Rich 2010; Marino et al. 2011a,b; Bresolin 2013) underscore this conclusion. The prolonged build-up of the peripheral zones of ETGs is also reflected in a radial decrease in the luminosityweighted stellar age, with $\sim 50 \%$ of the $V$-band luminosity contributed by stars younger than 5 Gyr (Gomes et al. 2015b, hereafter G15b). Circumstantial evidence for low-level SF in the outskirts of ETGs also comes from recent IFS studies (Papaderos et al. 2013, hereafter P13), which reveals that the $\mathrm{H} \alpha$ equivalent width $(E W(\mathrm{H} \alpha))$ of more than one half of ETGs increases outwardly.

In the light of such results, a representative census of SF patterns in ETGs appears to be of considerable interest because it may hold clues to the structural evolution of these systems and the role of intrinsic and environmental properties on the transformation of S0 into E galaxies. For example, a full understanding of the Butcher-Oemler (Butcher \& Oemler 1978) effect in galaxy clusters requires understanding of how the gradual cessation of SF activity and photometric dimming of the progenitor disk component is synchronized with the growth of the bulge and how this leads to a high bulge-to-total ratio $(\mathrm{B} / \mathrm{T})$ presentday ETG. Johnston et al. (2015) envisage a two-stage formation process for the bulge of S0 galaxies with the first phase completed early on and the second one fed by inflowing gas from the disk. This takes place in tandem with the starvation of SF activity in the latter as the result of ram pressure stripping. On the other hand, the evolutionary pathways of ETGs in lower-density environments are less clear, leaving space for various channels for the morphological $\mathrm{S} 0 \rightarrow \mathrm{E}$ transition.

Regardless of the environmental properties of present-day ETGs and of whether their SF activity is viewed from the perspective of quenching or rejuvenation that is possibly driven by minor merges (e.g., Mapelli et al. 2015), it is important to explore how the amplitude and spatial properties of low-level SF in these systems are related to their gas mass fraction and B/T ratio, how the SFR surface density and stellar initial mass function (IMF) change as the cold-gas supply is gradually depleted, and what intrinsic properties determine whether SF is spatially organized in contiguous rims or spiral-arm-like patterns, rather than in a diffuse and/or patchy collection of faint HII regions.

IFS studies can obviously add key insights into this subject, permitting simultaneous analysis of SF patterns and kinematics at the interface between S0 and E galaxies. A superb observational foundation for this is now provided by the Calar Alto Legacy Integral Field Area survey (CALIFA, Sánchez et al. 2012) - the first IFS survey to map the entire optical spectral range and spatial extent of a representative sample of the Hubble sequence in the local Universe.

Here we report on the detection of faint star-forming spiralarm-like features in three ETGs. The galaxies we study are similar to type i ETGs - systems characterized by a nearly constant $E W(\mathrm{H} \alpha) \leq 3 \AA$ throughout their extranuclear component (P13) - with the additional feature of a steep $E W(\mathrm{H} \alpha)$ increase in their outermost periphery. This led us in Gomes et al. (2015a) to assign them the notation i+ and motivated a more detailed analysis here. In Sect. 2 we briefly comment on the general properties of the i+ ETGs and in Sect. 3 demonstrate, through a combined analysis of SDSS imaging and CALIFA IFS data, that the $E W(\mathrm{H} \alpha)$ excess in the periphery of these systems comes from low-level SF that is associated with an ongoing insideout growth process. The main results and conclusions from this study are summarized in Sect. 4.

\section{Data and general description}

The ETGs discussed here, NGC 1167 (SA0), NGC 1349 (S0) and NGC 3106 (S0), at a distance of, respectively, 66.2, 87.7 and $90.1 \mathrm{Mpc}$, according to $\mathrm{NED}^{1}$, were included in a statistical study by P13 with the main focus on the radial distribution of the $E W(\mathrm{H} \alpha)$ and diagnostic emission-line ratios after Baldwin et al. (1981, hereafter BPT), followed by a more detailed 2D analysis of their nebular and stellar component in G15b. The second brightest member of a group of six normal-luminosity $\left(M_{B} \simeq-20\right)$ galaxies is NGC 1167, whereas NGC 1349 can be considered truly isolated, since they lack any bright companion within $\sim 500 \mathrm{kpc}$, according to NED. As for NGC 3106, it is a field galaxy with seven dwarfish $\left(-18.5 \lessgtr M_{\mathrm{r}} \lesssim-15.1\right)$ companions listed in NED, with a linear and a velocity separation of $0.3-10.2 \mathrm{kpc}$ and $\leq 100 \mathrm{~km} \mathrm{~s}^{-1}$, respectively. An in-depth study of these low-mass companions, two of which display emissionline spectra, will be presented in Kehrig et al. (in prep.). With the exception of NGC 1167, classified as a Seyfert, as also evident from its prominent radio jet, the nuclear spectra of these type i+ ETGs show faint nebular emission $(E W(\mathrm{H} \alpha) \leq 3 \AA)$ and LINER BPT ratios within their effective radius $R_{\text {eff }}(\mathrm{G} 15 \mathrm{~b})$. With regard to their photometric structure, the ETGs under study qualify by their bulge Sérsic index and bulge-to-total $(\mathrm{B} / \mathrm{T})$ ratio ( $\eta \lesssim 2$ and $\leq 1 / 3$, respectively; Breda et al., in prep., hereafter B15) as pseudo-bulge galaxies. Besides SDSS imaging data, this study uses low-spectral resolution $(R \sim 850)$ IFS CALIFA data with PMAS/PPaK (Roth et al. 2005; Kelz et al. 2006) reduced with the CALIFA data processing pipeline v1.5 (García-Benito et al. 2015). Emission-line flux and equivalent width maps were

https://ned.ipac.caltech.edu/ 

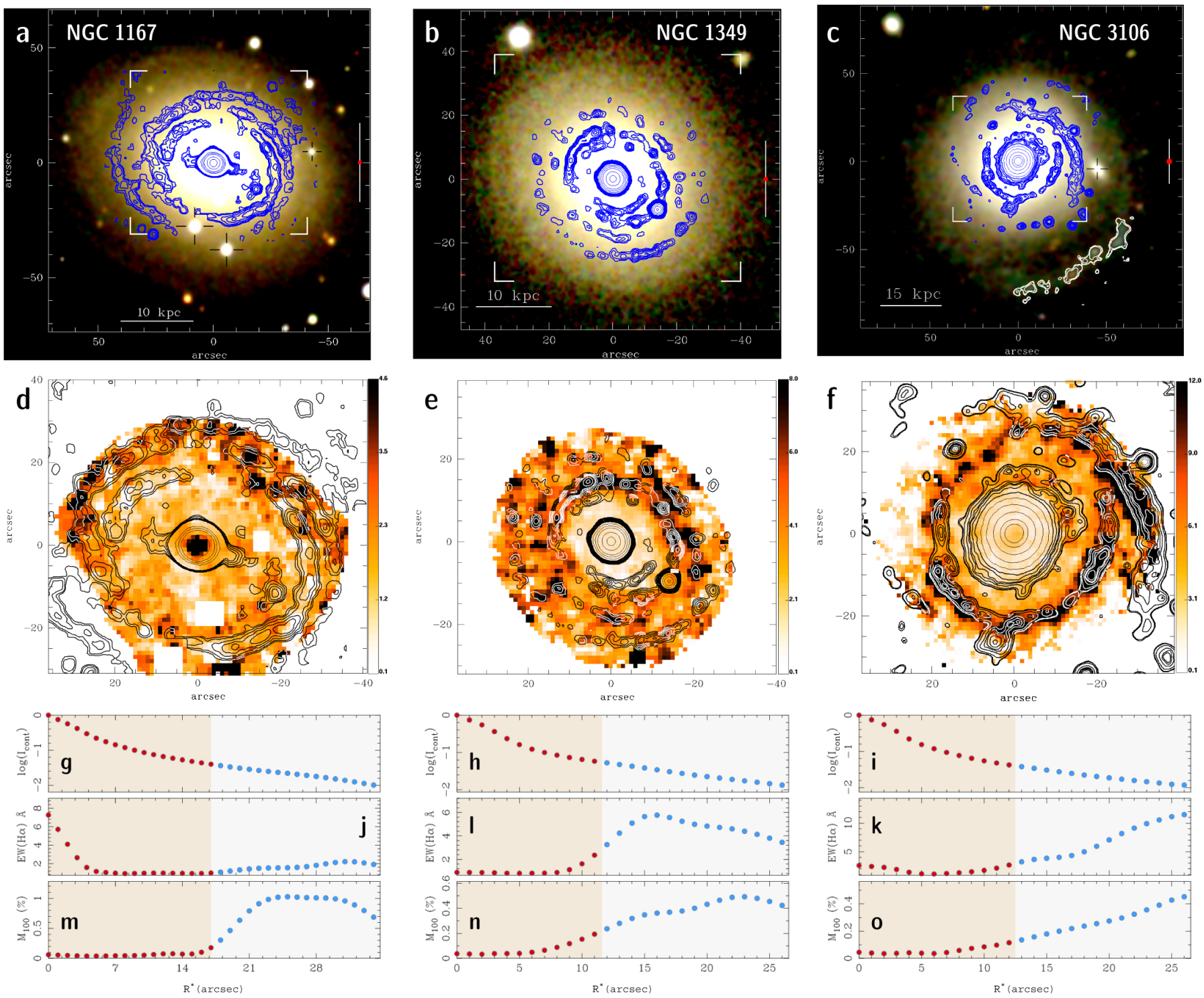

Fig. 1. a)-c): True-color SDSS image composites of the type i+ ETGs under study. The overlaid contours depict the morphology of the spiral-like star-forming features, as revealed by applying an unsharp-masking technique. The outermost contour corresponds to a surface brightness level $\mu_{r} \simeq 25 \mathrm{mag} / \square^{\prime \prime}$ for NGC 1167 and $\simeq 26 \mathrm{mag} / \square^{\prime \prime}$ for NGC 1349 and NGC 3106 , and the vertical line to a linear scale of $2 R_{\text {eff }}$ in the SDSS $r$ band. Bright foreground stars (panel a)) and a dwarf galaxy at the redshift of NGC 3106 (panel c)) are shown with crosses, and the edges of the CALIFA field of view are delineated in white color. $\mathbf{d})-\mathbf{f}): E W(\mathrm{H} \alpha)$ maps, as derived from CALIFA IFS data, overlaid with the same contours as in panels a)-c). Radial profiles of the intensity of the stellar continuum, normalized to its central value (g)-i)), $E W(\mathrm{H} \alpha)(\mathbf{j})-\mathbf{k}))$ and mass fraction $M_{100}(\%)$ of stars younger than $\left.100 \mathrm{Myr}(\mathbf{m})-\mathbf{o}\right)$ ).

computed with the Porto3D pipeline (Kehrig et al. 2012, hereafter K12; P13; see G15b for a full description of its latest version), which allows for spaxel-by-spaxel spectral fitting with STARLIGHT (Cid Fernandes et al. 2005) and subtraction of the best-fitting stellar spectrum from the observed one, thereby permitting accurate extraction of the pure nebular emission.

\section{Spiral-like SF features in the ETG periphery}

Figure 1 gives a synoptic view of the photometric and spectroscopic evidence for the presence of spiral-like star-forming patterns in the three i+ ETGs. Already visual inspection of SDSS poststamp images reveals traces of faint bluish patches and/or ripples in their low-surface brightness (LSB) extranuclear component. After a flux-conserving unsharp masking technique (Papaderos et al. 1998) on SDSS broadband data, these subtle patterns become clearly discernible as contiguous spiral-like features (contours in panels a-c) over a linear extent of $20-40 \mathrm{kpc}$, in close resemblance to spiral arms in normal latetype galaxies. Because of their faintness $\left(24 \lesssim \mu_{r}\left(\mathrm{mag} / \square^{\prime \prime}\right) \lesssim\right.$ 26 ), the detected structures contribute on average less than $10 \%$ of the local line-of-sight intensity, thereby leaving only subtle signatures on color maps and radial color profiles (see B15 for details). Likewise, their integrated magnitude $(-16 \lesssim$ $\left.M_{\mathrm{r}} \lesssim-18\right)$ translates to a negligible $(<2 \%)$ fraction of the total galaxy luminosity. Whereas in NGC 1349 these features protrude slightly inwards of $R_{\text {eff }}\left(11^{\prime \prime} .6\right)$ and extend over a radial zone $0.7 \leq R_{\text {eff }} \leq 2.3$, in the case of NGC 1167 and NGC 3106 they are detected well outside the nuclear region $\left(1.2-2.6 R_{\mathrm{eff}}\right.$ with $R_{\text {eff }}=17^{\prime \prime}$ and 1.5-6.2 $R_{\text {eff }}$ with $R_{\text {eff }}=12^{\prime \prime}$, , respectively). This is to be contrasted with several late-type pseudobulge galaxies (e.g., Kormendy \& Kennicutt 2004) and the S0 
NGC 6762 where K12 found through application of the same unsharp-masking technique, faint spiral features to be exclusively embedded within the pseudobulge $\left(<R_{\mathrm{eff}}\right)$.

In panels $\mathrm{d}-\mathrm{f}$ of Fig. 1 the spiral-like features revealed by unsharp-masking (contours) are shown overlaid with $E W(\mathrm{H} \alpha)$ maps obtained from CALIFA IFS data. It can be seen that the former are spatially correlated with more extended zones of enhanced $E W(\mathrm{H} \alpha)$, which implies that they are not purely stellar relics from fading spiral arms that have long ceased forming stars, but instead sites of ongoing SF. Evidently, the morphology of the detected LSB features is hardly compatible with the interpretation of them arising from shock excitation due to, e.g., infalling gas, or ionization by a central AGN. Likewise, the hypothesis of photoinization by the (evenly distributed) pAGB stellar background can be discarded on morphological grounds. Another argument rests on the $E W(\mathrm{H} \alpha)$ of these spiral-like structures, determined to be $2.6-5 \AA$ in NGC 1167 and reaching to $>20 \AA$ in NGC 3106: As shown in, e.g., Binette et al. (1994), Cid Fernandes et al. (2011) and, in more detail, in G15b, pAGB photoinization can account for a maximum $E W(\mathrm{H} \alpha) \simeq 2.4 \AA$ $\left(\equiv \mathrm{EW}_{\star}^{+}\right)$for a broad range of stellar metallicities and IMFs. The fact that the $E W(\mathrm{H} \alpha)$ exceeds $\mathrm{EW}_{\star}^{+}$, rules out pAGB photoionization as the physical origin of the detected spiral-like features.

The outer rise of the $E W(\mathrm{H} \alpha)$ above $\mathrm{EW}_{\star}^{+}$can also be appreciated from the radial profiles (panels $\mathrm{j}-\mathrm{k}$ ). These were derived with the isophotal annuli (isan) technique (Papaderos et al. 2002, see K12, P13 and G15b for applications to IFS data), whereby the emission-line free stellar continuum between $6390 \AA$ and $6490 \AA$ (panels g-i) was used for the isan definition. It can be seen that in all cases the mean $E W(\mathrm{H} \alpha)$ increases smoothly beyond $R_{\text {eff }}$ (shaded area) and reaches in the case of NGC 1349 and NGC 3106 values between $\sim 6 \AA$ and $\gtrsim 12 \AA$.

In summary, the combined analysis of SDSS imaging and CALIFA IFS data consistently indicates that the LSB spirallike features in the extra-nuclear component of the three type i+ ETGs under study are sites of ongoing SF. It should be pointed out that the presence of HII regions in the periphery of our ETGs has previously been noticed in the course of an investigation of metallicity gradients in the CALIFA galaxy sample (Sánchez 2013; Sánchez et al. 2014), even though not given a detailed discussion owing to the scope and statistical nature of those studies. Also G15b show that the outer SF zone in type i+ ETGs appears on BPT diagrams to be very similar to HII regions in late-type galaxies and it is generally located in the "intermediate" zone between the empirical SF sequence by Kauffmann et al. (2003) and the "maximum SF" boundary from Kewley et al. (2001). Obviously, the detectability and spectroscopic BPT classification of such embedded spiral features depends on their intrinsic properties ( $\mathrm{H} \alpha$ and broad-band surface brightness) and the observational data used (e.g., spectroscopic aperture, continuum $\mathrm{S} / \mathrm{N})$.

Quantitatively, our results in G15b indicate that the SF zone in type i+ ETGs is discernible on radial BPT-ratio profiles only when it contains appreciable nebular emission, as in the case of, e.g., NGC 1349 and NGC 3106. The H $\alpha$ luminosity fraction associated with the spiral-like SF features increases from $\sim 26 \%$ in NGC 1167 to $68 \%$ in NGC 3106 , reaching up to $80 \%$ in NGC 1349. Despite their faintness on broadband images, they therefore provide a significant, if not the dominant fraction of the total $\mathrm{H} \alpha$ emission. Their $\mathrm{H} \alpha$ luminosity, corrected for the contribution of pAGB photoionization, translates by the Kennicutt (1989) calibration to a SFR between 0.08 and $0.32 M_{\odot} \mathrm{yr}^{-1}$. The latter yields for the $\mathcal{M}_{\star}$ determined by $\mathrm{G} 15 \mathrm{~b}$ $\left(1.5 \ldots 2.6 \times 10^{11} M_{\odot}\right)$, a very low $\left(\lesssim 10^{-12} \mathrm{yr}^{-1}\right)$ specific SFR. This value will increase by no more than $30 \%$ if intrinsic extinction is taken into account, given the only slightly enhanced $\mathrm{H} \alpha / \mathrm{H} \beta$ ratio (2.95-3.2) in the star-forming periphery. Hence, SFRs based on the $\mathrm{H} \alpha$ luminosity, even if sustained over $1 \mathrm{Gyr}$, would produce only a tiny $(\ll 1 \%)$ fraction of the present-day $\mathcal{M}_{\star}$. It is also noteworthy that, despite their low SFR surface density $\left(\lesssim 1 \times 10^{-3} M_{\odot} \mathrm{yr}^{-1} \mathrm{kpc}^{-2}\right), \sim 3$ times lower than the average value in the Milky Way and comparable to that in LSB dwarf irregulars (cf. Kennicutt \& Evans 2012), the detected spirallike features have to contain a high-mass tail of ionizing stars. This yields a potentially important observational constraint in the context of the ongoing debate on possible stochasticity effects (e.g., Cerviño \& Mas-Hesse 1994) and spatial variations in the IMF of massive ETGs (e.g., Cappellari et al. 2012).

A question of considerable interest is whether the LSB spiral-like patterns in i+ ETGs witness a brief rejuvenation episode that has been triggered by, e.g., capture of a gas-rich satellite, or, instead represent localized SF signatures of a spatially and temporally extended inside-out formation process. Various lines of evidence favor the second interpretation. For example, deep near-UV archival data with GALEX impressively reveals SF activity all over the extended LSB periphery of NGC 1167 . The massive $\left(1.8 \times 10^{10} M_{\odot}\right)$ HI halo of this galaxy that reaches out to $\sim 80 \mathrm{kpc}$ (Emonts et al. 2006), and the presence of extended HI reservoirs in about $1 / 4$ of mostly disky ETGs (Serra et al. 2012) suggest that a significant fraction of these seemingly "old and dead" systems have the gas supply to sustain SF and their continued growth. The ongoing insideout assembly of type i+ ETGs is also indicated by the outwardly increasing mass fraction of stars younger than $100 \mathrm{Myr}\left(M_{100}\right)$, which reaches mean values between $\sim 0.4 \%$ and $\sim 1 \%$ for $r \gtrsim$ $R_{\text {eff }}$ (Fig. 1, panels m-o). Assuming that in ETGs light roughly scales with stellar mass, these values translate to an increase by $2 \%-5 \%$ in the total $\mathcal{M}_{\star}$ over the past $1 \mathrm{Gyr}$, in agreement with previous evidence for a significant growth of $\mathcal{M}_{\star}$ since $z \sim 1$ (cf. Introduction). The tension between SFRs estimated from the $\mathrm{H} \alpha$ luminosity of the spiral-like features $\left(<1 M_{\odot} / \mathrm{yr}\right)$ and those obtained from spectral synthesis (a few $M_{\odot} /$ yr) suggest that Balmer-line emission may yield a lower bound to the total SFR of ETGs, presumably because of extensive Lyman continuum photon escape through the tenuous/porous gas component of ETGs (cf. discussion in P13). In addition, a spatially varying IMF, being fully populated at the locus of the spiral features and truncated at its ionizing high-mass end elsewhere, might offer a non-mutually exclusive alternative.

It is conceivable that type i+ ETGs have previously been forming stars at a much higher pace - a conjecture in qualitative agreement with the presence of ionized gas disks in intermediate-redshift ETGs (Jaffé et al. 2014) - and are now seen in an advanced stage of a prolonged, gradually declining SF process (e.g., Kaviraj et al. 2007). The Gyr-long inside-out growth of ETGs is also suggested by the outwardly increasing luminosity contribution of stellar populations younger than 5 Gyr (G15b) and their by $\sim 0.1-0.25$ dex lower light-weighted age in their periphery (e.g., González Delgado et al. 2015), following a general trend for Hubble-type galaxies (e.g., Pérez et al. 2013). The observed size evolution of quiescent galaxies at $z \sim 2$ to present-day ETGs (e.g., Daddi et al. 2005; van Dokkum et al. 2008) is also consistent with a gradual inside-out assembly process.

Such considerations, and the observational evidence presented here, support the view that the subtle spiral-like SF features detected in type i+ ETGs through unsharp-masking 
primarily reflect a continuous inside-out $\mathcal{M}_{\star}$ growth process. Even though these systems may experience a transient rejuvenation through, e.g., accretion of a gas-rich satellite, this process alone appears incapable of accounting for various observational constraints, including the smooth radial increase of the mass fraction $M_{100}$ of stars younger than $100 \mathrm{Myr}$.

The observed spatial segregation of the nebular component of i+ ETGs in two spectroscopically distinct radial zones - an inner SF-devoid zone that contains faint LINER emission and an outer star-forming zone with strong nebular emission - calls for an examination of possible aperture effects in studies of such galaxies and their morphological analogs. For example, Pracy et al. (2014) report the presence of ongoing SF in the outskirts of two $\mathrm{E}+\mathrm{A}$ post-starburst galaxies and point out that the previously lacking evidence for SF-activity in these systems can be explained by an aperture bias in single-fiber spectroscopic studies. Likewise, an analogous yet inverse core-envelope spectrophotometric segregation is typical among low-mass star-forming galaxies (e.g., blue compact dwarfs or "green peas") where starburst activity is confined to the central part of an older stellar host (e.g., Papaderos et al. 1996; Gil de Paz et al. 2003; Amorín et al. 2012).

\section{Summary and conclusions}

We report on the detection of faint $\left(\mu \simeq 24-26 \mathrm{mag} / \square^{\prime \prime}\right)$, extended (20-40 kpc in diameter) spiral-arm-like features in the periphery $\left(R^{\star} \gtrsim R_{\text {eff }}\right.$ ) of three early-type galaxies (ETGs) through application of an unsharp-masking technique on SDSS imaging data. An analysis of integral field spectroscopy data from the CALIFA survey reveals a tight spatial correlation of these subtle spiral-like features with a broader zone of enhanced ( $3 \AA \lesssim E W(\mathrm{H} \alpha) \lesssim 20 \AA$ ) nebular emission in the ETG outskirts, which implies that they are not purely stellar relics from fading spiral arms that have long ceased forming stars, but sites of ongoing low-level star formation (SF).

The $\mathrm{H} \alpha$ luminosity of the detected spiral-like features translates into a star formation rate of $\sim 0.1-0.3 M_{\odot} / \mathrm{yr}$ and a very low $\left(\sim 10^{-12} \mathrm{yr}^{-1}\right)$ specific SFR. These estimates are significantly lower than those obtained from spectral synthesis models, which indicate that the i+ ETGs under study have assembled 2\%-5\% of their total stellar mass over the past one Gyr in an inside-out SF process. We argue that this discrepancy may be due to extensive Lyman continuum photon escape or a spatially varying IMF.

Despite their faintness, the detected star-forming spiral-armlike features are of considerable interest as they document the still ongoing growth of seemingly "old and dead" ETGs out of a cold-gas reservoir in their outskirts and could add valuable observational insights into the origin and stability of spiral structure in galaxy spheroids.

All but one of the studied ETGs, classified as type i+, display faint $(E W(\mathrm{H} \alpha) \simeq 1 \AA)$ LINER emission in their central part $\left(R^{\star} \leq 10^{\prime \prime}\right)$ and a spatial confinement of up to $80 \%$ of their integral $\mathrm{H} \alpha$ emission beyond the effective radius. This spatial segregation of the nebular component of $\mathrm{i}+$ ETGs in two spectroscopically distinct radial zones - an inner SF-devoid zone that contains faint LINER emission and an outer star-forming zone with strong nebular emission - calls for an examination of possible aperture effects in studies of such galaxies, and their morphological analogs, with single-fiber spectroscopic data.

Acknowledgements. This paper is based on data from the Calar Alto Legacy Integral Field Area Survey, CALIFA (http://califa.caha.es), funded by the
Spanish Ministery of Science under grant ICTS-2009-10, and the Centro Astronómico Hispano-Alemán. J.M.G. acknowledges support by Fundação para a Ciência e a Tecnologia (FCT) through the Fellowship SFRH/BPD/66958/2009 and POPH/FSE (EC) by FEDER funding through the program Programa Operacional de Factores de Competitividade (COMPETE). P.P. is supported by FCT through the Investigador FCT Contract No. IF/01220/2013 and POPH/FSE (EC) by FEDER funding through the program COMPETE. J.M.G. and P.P. acknowledge support by FCT under project FCOMP-01-0124-FEDER029170 (Ref. PTDC/FIS-AST/3214/2012), funded by FCT-MEC (PIDDAC) and FEDER (COMPETE) and the exchange programme "Study of EmissionLine Galaxies with Integral-Field Spectroscopy" (SELGIFS, FP7-PEOPLE2013-IRSES-612701), funded by the EU through the IRSES scheme. S.F.S. acknowledges support from CONACyT-180125 and PAPIIT-IA100815 grants. Support for L.G. is provided by the Ministry of Economy, Development, and Tourism's Millennium Science Initiative through grant IC120009, awarded to The Millennium Institute of Astrophysics, MAS. L.G. acknowledges support by CONICYT through FONDECYT grant 3140566. C.J.W. acknowledges support through the Marie Curie Career Integration Grant 303912. I.M. acknowledges financial support by the Junta de Andalucía through project TIC114, and the Spanish Ministry of Economy and Competitiveness (MINECO) through projects AYA2010-15169 and AYA2013-42227-P. R.A. Marino is funded by the Spanish program of International Campus of Excellence Moncloa (CEI). This research made use of the NASA/IPAC Extragalactic Database (NED) which is operated by the Jet Propulsion Laboratory, California Institute of Technology, under contract with the National Aeronautics and Space Administration.

\section{References}

Amorín, R., Pérez-Montero, E., Vílchez, J. M., Papaderos, P. 2012, ApJ, 749, 185

Annibali, F., Bressan, A., Rampazzo, R., et al. 2010, A\&A, 519, A40

Baldry, I. K., Robotham, A. S. G., Hill, D. T., et al. 2010, MNRAS, 404, 86

Baldwin, J. A., Phillips, M. M., \& Terlevich, R. 1981, PASP, 93, 5

Binette, L., Magris, C. G., Stasińska, G., \& Bruzual, A. G. 1994, A\&A, 292, 13 Bresolin, F. 2013, ApJ, 772, L23B

Butcher, H., \& Oemler, A.Jr. 1978, ApJ, 219, 18

Cappellari, M., McDermid, R., Alatalo, K., et al. 2012, Nature, 484, 485

Cerviño, M., \& Mas-Hesse, J. M. 1994, A\&A, 284, 749

Cid Fernandes, R., Mateus, A., Sodré, L., Stasińska, G., \& Gomes, J. M. 2005, MNRAS, 358, 363

Cid Fernandes, R., Stasińska, G., Mateus, A., et al. 2011, MNRAS, 413, 1687

Daddi, E., Renzini, A., Pirzkal, N., et al. 2005, ApJ, 626, 680

Demoulin-Ulrich, M., Butcher, H. R., \& Boksenberg, A. 1984, ApJ, 285, 527

Dopita, M. A., \& Sutherland, R. S. 1995, ApJ, 455, 468

Emonts, B. H. C., Morganti, R., Oosterloo, T. A., et al. 2006, Astron. Nachr., 327,139

Fang, J. J., Faber, S. M., Salim, S., Graves, G. J., \& Rich, R. M. 2012, ApJ, 761, 23

Finkelman, I., Brosch, N., Funes, J. G., Kniazev, A. Y., \& Väisänen, P. 2010, MNRAS, 407, 2475

García-Benito, R., Zibetti, S., Sánchez, S. F., et al. 2015, A\&A, 576, A135

Gil de Paz, A., Madore, B. F., Pevunova, O. 2003, ApJS, 147, 29

Gil de Paz, A., Boissier, S., Madore, B. F., et al. 2007, ApJS, 173, 185

Gomes, J. M., Papaderos, P., Kehrig, C. et al. 2015a, in Galaxies in 3D across the Universe, ed. Ziegler et al., 105 (G15a)

Gomes, J. M., Papaderos, P., Kehrig, C. et al. 2015b, A\&A, submitted (G15b)

González Delgado, R. M., García-Benito, R., Pérez, E., et al. 2015, A\&A, 581, A103

Ho, L. C. 2008, ARA\&A, 46, 475

Huang S., \& Gu, Q.-S. 2009, MNRAS, 398, 1651

Iglesias-Páramo, J., Vílchez, J. M., Galbany, L., et al. 2013, A\&A, 553, L7

Jaffé, Y. L., Aragón-Salamanca, A., Ziegler, B. L., et al. 2014, MNRAS, 440, $3491 \mathrm{~J}$

Johnston, E. J., Aragón-Salamanca, A., Merrifield, M. R., et al. 2015, in Galaxies in 3D across the Universe, eds. Ziegler et al., 225

Kauffmann, G., Heckman, T. M., Tremonti, C., et al. 2003, MNRAS, 346, 1055

Kaviraj, S., Schawinski, K., Devriendt, J. E. G., et al. 2007, ApJS, 173, 619

Kaviraj, S., Khochfar, S., Schawinski, K., et al. 2008, MNRAS, 388, 67

Kehrig, C., Monreal-Ibero, A., Papaderos, P., et al. 2012, A\&A, 540, A11 (K12)

Kelz, A., Verheijen, M. A. W., Roth, M. M. et al. 2006, PASP, 118, 129

Kennicutt, R. 1989, ApJ, 344, 685

Kennicutt, R. C., \& Evans, N. J. 2012, ARA\&A, 50, 531

Kewley, L. J., Dopita, M. A., Sutherland, R. S., Heisler, C. A., Trevena, J. 2001, ApJ, 556, 121

Kim, D. 1989, ApJ, 346, 653

Ko, J., Hwang, H. S., Im, M., et al. 2014, ApJ, 791, 134

Kormendy, J., \& Kennicutt, R. C. 2004, ARA\&A, 42, 603

Kulkarni, S., Sahu, D. K., Chaware, L., et al. 2014, New Astron., 30, 51 
Mapelli, M., Rampazzo, R., Marino, A. 2015, A\&A, 575, A16 Marino, R. A., Rosales-Ortega, F. F., Sánchez, S. F., et al. 2013, A\&A, 559, A114

Marino, A., Rampazzo, R., Bianchi, L., et al. 2014a, MNRAS, 411, 311

Marino, A., Bianchi, L., Rampazzo, R., et al. 2014b, ApJ, 736, 15

Pan, Z., Jinrong, L., Weipeng, L., et al. 2014, ApJ, 792, 1

Papaderos, P., Loose, H.-H., Fricke, K. J., Thuan, T. X. 1996, A\&A, 314, 59

Papaderos, P., Izotov, Y. I., Fricke, K. J., Thuan, T. X., \& Guseva, N. G. 1998 A\&A, 338, 43

Papaderos, P., Izotov, Y. I., Thuan, T. X., et al. 2002, A\&A, 393, 461

Papaderos, P., Gomes, J. M., Vílchez, J. M., et al. 2013, A\&A, 555, L1 (P13)

Pérez, E., Cid Fernandes, R., González Delgado, R. M., et al. 2013, ApJ, 764, L1P

Petty, S. M., Neil, J. D., Jarrett, T. H., et al. 2013, AJ, 146, 4

Phillips, M. M., Jenkins, C. R., Dopita, M. A., et al. 1986, AJ, 91, 1062

Pracy, M. B., Owers, M. S., Zwaan, M. et al. 2014, MNRAS, 443, 388P

Rampazzo, R., Marino, A., Tantalo, R., et al. 2007, MNRAS, 381, 245

Roth, M. M., Kelz, A., Fechner, T. et al. 2005, PASP, 117, 620

Salim, S., \& Rich, R. M. 2010, ApJ, 714, L290
Salim, S., Fang, J. J., Rich, R. M., et al. 2012, ApJ, 755, 2

Sánchez, S. 2013, Adv. Astron., 2013, 596501

Sánchez, S. F., Kennicutt, R. C., Gil de Paz, A., et al. 2012, A\&A, 538, A8

Sánchez, S. F., Rosales-Ortega, F. F., Iglesias-Páramo, J., et al. 2014, A\&A, 563, 49

Sarzi, M., Falcón-Barroso, J., Davies, R. L., et al. 2006, MNRAS, 366, 1151

Sarzi, M., Shields, J. C., Schawinski, K., et al. 2010,MNRAS, 402, 2187

Schawinski, K., Thomas, D., Sarzi, M., et al. 2007, MNRAS, 382, 1415

Schawinski, K., Lintott, C., Thomas, D., et al. 2009, AAS, 41, 277

Serra, P., Oosterloo, T., Morganti, R., et al. 2012, MNRAS, 422, 1835

Shapiro, K. L., Falcón-Barroso, J., van de Ven, G., et al. 2010, MNRAS, 402, 2140

Singh, R., van de Ven, G., Jahnke, K., et al. 2013, A\&A, 558, A43

Stasińska, G., Vale Asari, N., Cid Fernandes, R., et al. 2008, MNRAS, 391, L29

Trager, S. C., Faber, S. M., Worthey, G., González, J. J. 2000, AJ, 120, 165

Trinchieri, G., \& di Serego Alighieri, S. 1991, AJ, 101, 1647

van Dokkum, P., Franx, M., Kriek, M., et al. 2008, ApJ, 677, L5

Yan, R., \& Blanton, M. R. 2012, ApJ, 747, 61 